\title{
Mise à jour des Recommandations canadiennes provisoires concernant l'utilisation d'une dose fractionnée du vaccin contre la fièvre jaune durant une pénurie du vaccin
}

Groupe de travail sur la fièvre jaune au nom du Comité consultatif de la médecine tropicale et la médecine des voyages (CCMTMV)*

\section{Mise à jour de septembre 2016}

\begin{abstract}
Les Recommandations canadiennes provisoires concernant l'utilisation d'une dose fractionnée du vaccin contre la fièvre jaune durant une pénurie du vaccin (1), publiées au mois d'août 2016, ont été rédigées par le Comité consultatif de la médecine tropicale et de la médecine des voyages (CCMTMV) afin de remédier à la récente pénurie de stocks de vaccin contre la fièvre jaune. Le fabricant homologué du vaccin contre la fièvre jaune au Canada a mis en $œ u v r e$ des mesures afin de remédier à cette pénurie. Les stocks habituels de vaccins sont désormais disponibles pour les centres de vaccination contre la fièvre jaune désignés. Les recommandations provisoires (1) doivent seulement être mises en œuvre durant une pénurie de vaccins. Le CCMTMV ne recommande plus d'utiliser une dose fractionnée du vaccin contre la fièvre jaune. Veuillez consulter les recommandations standard relatives à la vaccination contre la fièvre jaune dans le Guide canadien d'immunisation (2), et dans la Déclaration sur la fièvre jaune à l'intention des voyageurs du CCMTMV (3).
\end{abstract}

Citation proposée : Groupe de travail sur la fièvre jaune au nom du Comité consultatif de la médecine tropicale et la médecine des voyages (CCMTMV). Mise à jour des Recommandations canadiennes provisoires concernant I'utilisation d'une dose fractionnée du vaccin contre la fièvre jaune durant une pénurie du vaccin. Relevé des maladies transmissibles au Canada 2016;42:209. https://doi.org/10.14745/ccdr.v42i09a05f

\section{Références}

1. Groupe de travail sur la fièvre jaune, au nom du Comité consultatif de la médecine tropicale et de la médecine des voyages (CCMTMV). Recommandations canadiennes provisoires concernant l'utilisation d'une dose fractionnée du vaccin contre la fièvre jaune durant une pénurie du vaccin. Relevé des maladies transmissibles au Canada 2016;42:158-60. http://www.phac-aspc.gc.ca/publicat/ccdrrmtc/16vol42/dr-rm42-8/ar-02-fra.php.

2. Agence de la santé publique du Canada. Guide canadien d'immunisation. Partie 4 : Vaccins actifs, vaccin contre la fièvre jaune de 2012. http://www.phac-aspc.gc.ca/publicat/ cig-gci/p04-yfev-fiej-fra.php.
3. Comité consultatif de la médecine tropicale et de la médecine des voyages (CCMTMV). Déclaration sur la fièvre jaune à l'intention des voyageurs. Relevé des maladies transmissibles au Canada, 2013;39:1-18. http://www.phacaspc.gc.ca/publicat/ccdr-rmtc/13vol39/acs-dcc-2/index-fra. php. 